\title{
Changes in Cortical Thickness in Patients With Early Parkinson's Disease at Different Hoehn and Yahr Stages
}

\author{
Yuyuan Gao ${ }^{1 \dagger}$, Kun Nie ${ }^{1 \dagger}$, Mingjin Mei', Manli Guo', Zhiheng Huang', Limin Wang', \\ Jiehao Zhao', Biao Huang', Yuhu Zhang ${ }^{1 *}$ and Lijuan Wang ${ }^{1 *}$ \\ 'Department of Neurology, Guangdong General Hospital, Guangdong Academy of Medical Sciences, Guangdong \\ Neuroscience Institute, Guangzhou, China, ${ }^{2}$ Department of Radiology, Guangdong General Hospital, Guangdong Academy \\ of Medical Sciences, Guangzhou, China
}

OPEN ACCESS

Edited by:

Muthuraman Muthuraman

University Medical Center of the

Johannes Gutenberg University

Mainz, Germany

Reviewed by:

Dumitru Ciolac,

Nicolae Testemiţanu State University of Medicine and Pharmacy, Moldova

Gabriel Gonzalez-Escamilla,

Johannes Gutenberg University

Mainz, Germany

*Correspondence:

Yuhu Zhang

yhzhangsd@126.com

Lijuan Wang

wljgd68@163.com

tThese authors have contributed equally to this work

Received: 09 April 2018 Accepted: 07 November 2018

Published: 27 November 2018

Citation:

Gao Y, Nie K, Mei M, Guo M, Huang $Z$, Wang $L$, Zhao J, Huang $B$, Zhang $Y$ and Wang $L$ (2018) Changes in Cortical Thickness in Patients With Early Parkinson's Disease at Different

Hoehn and Yahr Stages.

Front. Hum. Neurosci. 12:469. doi: 10.3389/fnhum.2018.00469
Objectives: This study was designed to explore changes in cortical thickness in patients with early Parkinson's disease (PD) at different Hoehn and Yahr $(H-Y)$ stages and to demonstrate the association of abnormally altered brain regions with part III of the Unified Parkinson's Disease Rating Scale (UPDRS-III).

Materials and Methods: Sixty early PD patients and 29 age- and gender-matched healthy controls (HCs) were enrolled in this study. All PD patients underwent comprehensive clinical and neuropsychological evaluations and $3.0 \mathrm{~T}$ magnetic resonance scanning. Patients with $\mathrm{H}-\mathrm{Y}$ stage $\leq 1.5$ were included in the mild group, and all other patients were included in the moderate group. FreeSurfer software was used to calculate cortical thickness. We assessed the relationship between UPDRS-III and regional changes in cortical thinning, including the bilateral fusiform and the temporal lobe.

Results: The average cortical thickness of the temporal pole, fusiform gyrus, insula of the left hemisphere and fusiform gyrus, isthmus cingulate cortex, inferior temporal gyrus, middle temporal cortex and posterior cingulate cortex of the right hemisphere exhibited significant decreasing trends in HCs group and PD groups (i.e., the mild group and moderate group). After controlling for the effects of age, gender, and disease duration, the UPDRS-III scores in patients with early PD were correlated with the cortical thickness of the left and right fusiform gyrus and the left temporal pole $(p<0.05)$.

Conclusion: The average cortical thickness of specific brain regions reduced with increasing disease severity in early PD patients at different $\mathrm{H}-\mathrm{Y}$ stages, and the UPDRS-III scores of early PD patients were correlated with cortical thickness of the bilateral fusiform gyrus and the left temporal pole.

Keywords: Parkinson's disease, Hoehn and Yahr stage, UPDRS part III, FreeSurfer, magnetic resonance imaging

Abbreviations: AD, Alzheimer's disease; ADL, activities of daily living; CT, computed tomography; DTI, diffusion tensor imaging; FOG, freezing of gait; GM, gray matter; HAMA, Hamilton Anxiety Scale; HAMD, Hamilton Depression Scale; HC, healthy control; H-Y stage, Hoehn and Yahr stage; MDS, movement disorder society; MMSE, mini mental state examination MoCA, montreal cognitive assessment; MRI, magnetic resonance imaging; NMS, non-motor symptoms; PD, Parkinson's disease; PDD, Parkinson's disease with dementia; PD-MCI, Parkinson's disease with mild cognitive impairment; PD-NC, Parkinson's disease with normal cognition; UPDRS-III, part III of the Unified Parkinson's Disease Rating Scale; WM, white matter. 


\section{INTRODUCTION}

Parkinson's disease is the second most common neurodegenerative disease, next only to $\mathrm{AD}$, and is characterized by motor symptoms, such as bradykinesia, rest tremor and muscle rigidity, and non-motor symptoms (NMS), such as cognitive impairment, constipation, anxiety, depression, and sleep disorders. The main pathological changes in PD are the loss of large quantities of dopaminergic neurons from the midbrain substantia nigra pars compacta and the formation of Lewy bodies by misfolded $\alpha$-synuclein, resulting in basal ganglia dysfunction (Kalia and Lang, 2015). The prevalence of $\mathrm{PD}$ is $1-2 \%$ among elderly over 65 years of age and $3-5 \%$ in those over 85 years of age (Alves et al., 2008). By 2030, there are expected to be five million PD patients in China (Dorsey et al., 2006). Two hundred years after the first documented description of PD (Kalia and Lang, 2015) and although the core pathological features of PD have been established, the related pathogenic mechanism of the misfolded $\alpha$-synuclein remains controversial (Kalaitzakis et al., 2008). In 2003, Braak et al. (2003) divided PD into six stages to describe the development of PD (Braak et al., 2003). According to Braak's staging system, the evolution of motor symptoms and NMS of PD can be well explained. The onset of motor symptoms is suggested to accompany the death of at least $50 \%$ of the dopaminergic neurons in the substantia nigra, corresponding to Braak stage III/IV. However, patients at Braak stages I and II mainly exhibit NMS, such as orthostatic hypotension, olfactory dysfunction and rapid eye movement (REM) sleep abnormalities. Therefore, early identification of preclinical and early-stage PD patients may aid in the early management of PD.

The Hoehn-Yahr (H-Y) scale was created in 1967 and is widely used in the evaluation of the clinical condition of PD (Hoehn and Yahr, 1967). The quality of life of PD patients is significantly reduced during disease development, whereby patients progress from modified H-Y stage $\leq 2.5$ to modified H-Y stage $>3$ (Evans et al., 2011). The guidelines for the diagnosis of PD in China define patients with $\mathrm{H}-\mathrm{Y}$ stage $\leq 2.5$ as early PD patients (Chen et al., 2016). PD patients at different stages present brain structural changes, but the related pathogenic mechanism and pathophysiological changes remain unclear. The preclinical stage of PD is very difficult to diagnose because the symptoms and signs of patients are not typical, and there are no significant changes in anatomical images, such as CT and MRI. However, PD patients gradually present clinical symptoms and signs with increasing $\mathrm{H}-\mathrm{Y}$ stages and exhibit the typical symptoms at middle and late stages, which are not difficult to diagnose. Therefore, the identification of early PD imaging signs is of great importance for the early diagnosis of PD. Changes in cortical thickness (Mak et al., 2015), GM volume (Jia et al., 2015) and cortical microstructure (Nurnberger et al., 2017), increased cortical and subcortical atrophy rate (Tessa et al., 2014) and loss of cortical gyrification (Sterling et al., 2016) have been reported, and some imaging signs even correlate with disease progression (Ibarretxe-Bilbao et al., 2011; Jubault et al., 2011). Specifically, cortical thickness can be used in PD disease staging and cognitive impairment assessment (Zarei et al., 2013). Therefore, we hypothesized that abnormal changes in cortical thickness may occur in early-stage PD patients with $\mathrm{H}-\mathrm{Y}$ stage $\leq 1.5$ and $\mathrm{H}-\mathrm{Y}$ stage $\geq 2$ and that these changes would correlate with the severity of the disease and UPDRS-III. Therefore, this study investigated the cortical thickness abnormalities of early PD patients at different $\mathrm{H}-\mathrm{Y}$ stages and the correlation between cortical thickness and UPDRS-III, providing imaging signs for the early identification of preclinical and early-stage PD patients.

\section{MATERIALS AND METHODS}

\section{Participants}

This study selected 89 subjects from a database of the Department of Neurology, Guangdong General Hospital (Guangzhou, China), including 60 patients with early stage PD and 29 gender-, age-, and education level-matched HCs. The diagnosis of PD was independently performed in accordance with the British Brain Bank PD diagnostic criteria by two neurologists who held positions of attending physicians. In addition, the PD patients underwent a series of assessments. The motor function in PD patients was assessed using UPDRS-III. The severity of PD was assessed using the H-Y stage. Depression in PD patients was evaluated using the 24-item HAMD. Anxiety in PD patients was assessed by the HAMA. The ADL of PD patients were evaluated using the 14-item ADL Scale. The cognitive function of PD patients was evaluated using the MoCA and the MMSE. All PD subjects included in this study met the following criteria: age $\geq 40$ years, early stage $\mathrm{PD}$ ( $\mathrm{H}-\mathrm{Y}$ stage $\leq 2.5$ ), and no severe depression or anxiety (HAMD $\leq 20$ points and HAMA $\leq 29$ points). According to the $\mathrm{H}-\mathrm{Y}$ stage score, early PD patients with $\mathrm{H}-\mathrm{Y}$ stage $\leq 1.5$ were included in the mild group, and those with $2.5 \leq \mathrm{H}-\mathrm{Y}$ stage $\geq 2$ were included in the moderate group. Structural MRI was acquired from 28 early PD patients in the mild group and 32 early PD patients in the moderate group (Figure 1). Healthy volunteers did not have any neurological or mental illness and did not take medications known to affect the dopaminergic system. Structural MRI was acquired from 29 healthy subjects in the HC group. All subjects provided written informed consent to the research protocol, which was approved by the Medical Ethics Committee of Guangdong General Hospital.

\section{Collection of Magnetic Resonance Imaging (MRI) Data}

A GE 3.0 T MR imaging system (GE Healthcare, Fairfield, United States) was used. The magnetic resonance data of all subjects were obtained by three-dimensional spoiled gradient recalled (3D-SPGR) sequences. During scanning, the subjects were positioned on a patient bed in the supine position. The head was fixed on the matrix coil with a sponge pillow and a helmet to minimize head movement, and earplugs were worn. High signal-to-noise ratio three-dimensional T1-weighted images were 


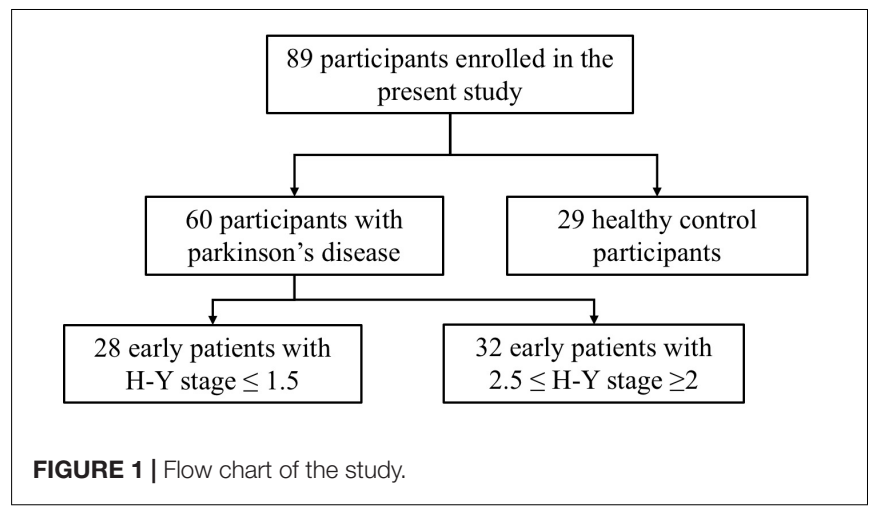

obtained by high-resolution scanning. The parameters were as follows: number of scanning slices, 146; slice thickness, $1.00 \mathrm{~mm}$; slice spacing, $1.00 \mathrm{~mm}$; matrix, $256 \times 256$; field of view (FOV), $512 \times 512$; voxel, $0.469 \times 0.469 \times 1.0 \mathrm{~mm}^{3}$; flip angle, $13^{\circ}$; repetition time (TR), $7.6 \mathrm{~ms}$; and echo time (TE), $3.3 \mathrm{~ms}$.

\section{Image Processing and Analysis}

MRIcron software was used to convert the MRI data of all subjects from DICOM format to NIFTI format, and the process of extracting cerebral cortex thickness was based on FreeSurfer v5.3.0 software ${ }^{1}$ on the Linux platform (ubuntu 14.04) according to previously published papers (Fischl and Dale, 2000; Fischl, 2012). For image preprocessing, the "recon-all" instruction was used, which included magnetic field inhomogeneity correction, motion correction, removal of non-brain tissues such as the scalp and skull, segmentation of the GM and WM boundaries, automatic topological correction and registration with standard brain templates, Gaussian smoothing, reconstruction of the bilateral cerebral cortex, and calculation of the cortical thickness. MNI coordinates were transformed from the Talairach

${ }^{1}$ http://surfer.nmr.mgh.harvard.edu coordinates by using the tool provided in http://imaging.mrccbu.cam.ac.uk/downloads/MNI2tal/tal2mni.m.

\section{Statistical Analysis}

SPSS 22.0 was used to analyze the data. A linear model (GLM) was defined for statistical analysis in the Qdec module of FreeSurfer. Brain areas with significant differences in cortical thickness, as determined by intergroup comparisons of the $\mathrm{HC}$, mild and moderate groups, were identified. For the $\mathrm{HC}$, mild and moderate groups, one-way ANOVA was used to compare age, the chisquare test was used to compare gender, and the Kruskal-Wallis $H$ test was used to compare education. For the mild and moderate groups, disease duration, ADL, HAMA, MMSE and H-Y stage were compared using the Mann-Whitney $U$ test, and UPDRS-III and MoCA were compared using a two-tailed $t$-test. Regression analysis of average cortical thickness of different brain areas and UPDRS-III scores using a general linear model while controlling for age, gender, and disease duration. A $p$-value $<0.05$ was considered statistically significant.

\section{RESULTS}

\section{Demographic and Clinical Characteristics of the HC, Mild and Moderate Groups}

Among the three groups, no significant differences in age $(F=1.742, p=0.181)$, gender $\left(\chi^{2}=3.620, p=0.164\right)$ and education $(H=0.048, p=0.976)$ were noted. Comparison of the mild and moderate groups revealed no significant differences in disease duration $(Z=-0.772, p=0.440)$, $\mathrm{ADL}(Z=-1.243$, $p=0.214)$, HAMD $(Z=-1.889, p=0.059)$, HAMA $(Z=-1.176$, $p=0.240)$, MoCA $(t=-0.022, p=0.983)$, MMSE $(Z=-1.381$, $p=0.167)$ and $\mathrm{H}-\mathrm{Y}$ stage $(Z=6.864, p=0.000)$, whereas significant differences in UPDRS-III $(t=-4.847, p<0.0001)$ were noted (Table $\mathbf{1})$.

TABLE 1 | Demographic and clinical data of subjects in the HC, mild, and moderate groups.

\begin{tabular}{|c|c|c|c|c|c|}
\hline Variables & HCs & Mild & Moderate & $F / H / \chi^{2} / t$ & $p$-value \\
\hline Age in years, mean (SD) & $59.17(1.88)$ & $57.32(1.65)$ & $61.59(1.39)$ & $1.742(F)$ & 0.181 \\
\hline Education in years, mean (SD) & $9.69(1.02)$ & $10.04(0.75)$ & $9.81(0.75)$ & $0.048(H)$ & 0.976 \\
\hline Disease duration, month, mean (SD) & - & 28.07 (3.34) & $30.44(6.37)$ & $-0.772(Z)$ & 0.440 \\
\hline UPDRS-III, mean (SD) & - & $19.29(1.60)$ & $31.08(1.79)$ & $-4.847(t)$ & $<0.0001$ \\
\hline HAMD & - & $4.93(0.73)$ & $7.41(0.91)$ & $-1.889(Z)$ & 0.059 \\
\hline HAMA & - & $4.57(0.71)$ & $5.88(0.75)$ & $-1.176(Z)$ & 0.240 \\
\hline MoCA, mean (SD) & - & $22.79(0.93)$ & $22.81(0.82)$ & $-0.022(t)$ & 0.983 \\
\hline MMSE, mean (SD) & - & $28.14(0.31)$ & $27.25(0.44)$ & -1.381 & 0.167 \\
\hline $\mathrm{H}-\mathrm{Y}$ stage & - & $1.5(1.0-1.5)$ & $2.25(2.0-2.5)$ & $6.864(Z)$ & 0.000 \\
\hline
\end{tabular}

$P D$ patients with $\mathrm{H}-Y$ stage $\leq 1.5$ were included in the mild group, and $P D$ patients with $2.5 \leq \mathrm{H}-Y$ stage $\geq 2$ were included in the moderate group.

ADL, activities of daily living; UPDRS-III, part III of the Unified Parkinson's Disease Rating Scale; HAMD, Hamilton Depression Scale; HAMA, Hamilton Anxiety Scale; MoCA, Montreal Cognitive Assessment; MMSE, Mini Mental State Examination; $\mathrm{H}-\mathrm{Y}$ stage, Hoehn and Yahr stage. Significant value is indicated in bold. 
TABLE 2 | Anatomical location of brain areas exhibiting significant differences in intergroup comparisons of the HC, mild and moderate groups.

\begin{tabular}{|c|c|c|c|c|c|c|c|c|c|c|}
\hline \multirow[t]{2}{*}{ Contrast } & \multirow[t]{2}{*}{ Regions } & \multirow[t]{2}{*}{ P-Max } & \multicolumn{3}{|c|}{ MNI coordinate } & \multirow{2}{*}{$\begin{array}{c}\text { Total surface } \\
\text { area size } \\
\left(\mathrm{mm}^{2}\right)\end{array}$} & \multirow[t]{2}{*}{ Vertices } & \multicolumn{3}{|c|}{ Mean thickness (mm) } \\
\hline & & & $x$ & $Y$ & $Z$ & & & HCs & Mild & Moderate \\
\hline \multirow{15}{*}{$\begin{array}{l}\text { Mild }>\text { Moderate } \\
p \text {-value }<0.001 \\
\text { Surface area } \\
\text { size }>100\end{array}$} & L temporal pole & 4.3345 & -34 & 10 & -39 & 165.28 & 302 & 2.249 & 2.125 & 1.777 \\
\hline & L superior frontal cortex & 4.0574 & -14 & 20 & 37 & 453.91 & 946 & 1.919 & 1.923 & 1.803 \\
\hline & $\mathrm{L}$ insula & 3.7611 & -29 & 17 & -8 & 455.60 & 1264 & 2.042 & 1.958 & 1.573 \\
\hline & $\mathrm{L}$ isthmus cingulate cortex & 3.5572 & -6 & -53 & 29 & 135.91 & 315 & 2.173 & 2.039 & 1.929 \\
\hline & L fusiform gyrus & 3.4439 & -37 & -53 & -25 & 296.43 & 411 & 1.946 & 1.849 & 1.564 \\
\hline & $\mathrm{R}$ inferior temporal cortex & 5.5224 & 52 & -53 & -22 & 332.07 & 574 & 2.101 & 2.088 & 1.833 \\
\hline & R lateral occipital cortex & 5.4167 & 45 & -71 & -13 & 417.44 & 572 & 1.730 & 1.736 & 1.608 \\
\hline & R fusiform cortex & 4.3463 & 40 & -44 & -27 & 452.93 & 828 & 1.929 & 1.830 & 1.486 \\
\hline & R posterior cingulate cortex & 4.2717 & 4 & -8 & 32 & 145.09 & 408 & 1.958 & 1.752 & 1.390 \\
\hline & R middle temporal cortex & 4.2589 & 54 & -4 & -35 & 763.49 & 1164 & 1.830 & 1.781 & 1.594 \\
\hline & R posterior cingulate cortex & 4.0841 & 8 & -10 & 44 & 209.59 & 524 & -- & -- & -- \\
\hline & $\mathrm{R}$ isthmus cingulate cortex & 3.9943 & 6 & -47 & 31 & 651.28 & 1511 & 2.197 & 2.004 & 1.735 \\
\hline & R superior parietal cortex & 3.7380 & 28 & -73 & 21 & 182.35 & 384 & 1.705 & 1.623 & 1.648 \\
\hline & R pars opercularis & 3.7155 & 36 & 18 & 14 & 314.43 & 947 & 1.790 & 1.647 & 1.490 \\
\hline & R superior frontal cortex & 3.7119 & 12 & 35 & 32 & 103.51 & 212 & 1.875 & 1.847 & 1.801 \\
\hline $\begin{array}{l}\text { Mild }<\text { Moderate } \\
p \text {-value }<0.001\end{array}$ & R superior frontal cortex & -3.7849 & 22 & 7 & 65 & 100.39 & 189 & -- & -- & -- \\
\hline \multirow{24}{*}{$\begin{array}{l}\text { HCs }>\text { Moderate } \\
p \text {-value }<0.001 \\
\text { Surface area } \\
\text { size }>100\end{array}$} & L temporal pole & 7.2720 & -33 & 10 & -39 & 340.30 & 691 & -- & -- & -- \\
\hline & $\mathrm{L}$ insula & 6.4471 & -31 & 13 & 7 & 1720.84 & 4517 & -- & -- & -- \\
\hline & L fusiform cortex & 6.2268 & -33 & -34 & -30 & 2075.62 & 3548 & -- & -- & -- \\
\hline & $\mathrm{L}$ isthmus cingulate cortex & 6.2197 & -7 & -39 & 35 & 5037.82 & 10788 & -- & -- & -- \\
\hline & L lateral occipital cortex & 5.1166 & -44 & -68 & -8 & 1324.11 & 2433 & 1.707 & 1.743 & 1.607 \\
\hline & L middle temporal cortex & 4.7842 & -54 & -10 & -31 & 929.54 & 1306 & 1.813 & 1.809 & 1.595 \\
\hline & $\mathrm{L}$ inferior parietal cortex & 4.6962 & -32 & -65 & 38 & 129.24 & 310 & 1.748 & 1.729 & 1.526 \\
\hline & L rostral middle frontal cortex & 4.6635 & -26 & 33 & 28 & 185.56 & 320 & 1.894 & 1.821 & 1.746 \\
\hline & L precentral cortex & 4.4666 & -41 & 1 & 27 & 295.74 & 703 & 1.592 & 1.624 & 1.630 \\
\hline & L inferior temporal gyrus & 3.9811 & -54 & -39 & -29 & 158.17 & 225 & 2.107 & 2.071 & 1.833 \\
\hline & L supramarginal gyrus & 3.7352 & -52 & -43 & 31 & 145.87 & 329 & 1.590 & 1.602 & 1.464 \\
\hline & L superior parietal cortex & 3.6853 & -27 & -77 & 16 & 290.15 & 533 & 1.683 & 1.653 & 1.660 \\
\hline & R precuneus & 7.7128 & 10 & -56 & 23 & 6221.94 & 13769 & 1.639 & 1.533 & 1.260 \\
\hline & R medial orbitofrontal & 6.1465 & 11 & 19 & -18 & 212.17 & 517 & 2.004 & 1.833 & 1.602 \\
\hline & R fusiform cortex & 5.8971 & 30 & -60 & -17 & 2229.04 & 3877 & -- & -- & -- \\
\hline & R postcentral gyrus & 5.2073 & 42 & -6 & 19 & 2128.36 & 5409 & 1.591 & 1.542 & 1.628 \\
\hline & R lateral orbitofrontal cortex & 4.9392 & 29 & 31 & -11 & 405.12 & 700 & 1.908 & 1.794 & 1.555 \\
\hline & $\mathrm{R}$ rostral middle frontal cortex & 4.8654 & 28 & 50 & 1 & 228.63 & 363 & 1.853 & 1.780 & 1.733 \\
\hline & R middle temporal cortex & 4.8144 & 54 & -12 & -31 & 1071.64 & 1639 & -- & -- & -- \\
\hline & R superior parietal cortex & 4.8007 & 30 & -72 & 19 & 303.46 & 615 & -- & -- & -- \\
\hline & R caudal middle frontal cortex & 4.4205 & 34 & 4 & 33 & 156.69 & 340 & 1.707 & 1.684 & 1.680 \\
\hline & R inferior temporal gyrus & 4.1356 & 51 & -22 & -35 & 176.07 & 277 & -- & -- & -- \\
\hline & R cuneus cortex & 4.0910 & 7 & -83 & 13 & 256.64 & 333 & 1.656 & 1.522 & 1.462 \\
\hline & R precentral gyrus & 3.7446 & 50 & 4 & 13 & 120.41 & 246 & 1.602 & 1.550 & 1.637 \\
\hline $\begin{array}{l}\text { HCs }>\text { Mild } p \text {-value } \\
<0.01\end{array}$ & L supramarginal gyrus & -3.2027 & -54 & -42 & 32 & 26.43 & 55 & -- & -- & -- \\
\hline
\end{tabular}

$P D$ patients with $\mathrm{H}$-Y stage $\leq 1.5$ were included in the mild group, and PD patients with $2.5 \leq \mathrm{H}$-Y stage $\geq 2$ were included in the moderate group. "- - " means that more than one cluster located on the same brain areas, and these different clusters only have one mean cortical thickness. The $X, Y$, and $Z$ coordinates refer to the anatomical location of brain areas. 


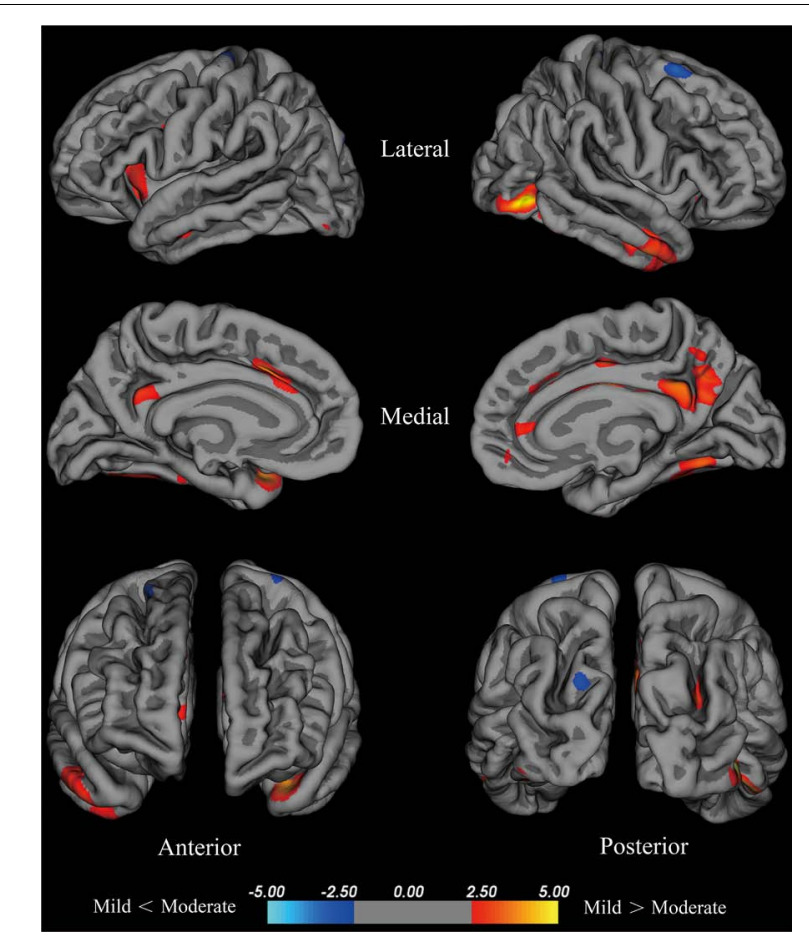

FIGURE 2 | Brain areas with significant cortical thickness differences between the mild group and the moderate group in the right and left hemispheres ( $p<0.001)$. PD patients with $\mathrm{H}-\mathrm{Y}$ stage $\leq 1.5$ were included in the mild group, and PD patients with $2.5 \leq \mathrm{H}-\mathrm{Y}$ stage $\geq 2$ were included in the moderate group. Red: mild > moderate, the closer the color is to yellow, the greater the difference. Blue: mild < moderate, the lighter the blue is, the larger the difference.

\section{Cortical Thickness Across the HC, Mild and Moderate Groups}

The coordinates, number of vertices, maximum $p$ value, extent of areas with differences in cortical thickness, and average cortical thicknesses of relevant brain areas can be found in Table 2 .

\section{Cortical Thickness of the Moderate Group Compared With the Mild Group}

Compared with the mild group, the moderate group exhibited large areas (size $\left.>100 \mathrm{~mm}^{2}\right)$ of significant decreases $(p<0.001)$ in the temporal pole, superior frontal cortex, insula, isthmus cingulate cortex, fusiform gyrus of the left hemisphere, inferior temporal cortex, lateral occipital cortex, fusiform cortex, posterior cingulate cortex, middle temporal cortex, posterior cingulate cortex, isthmus cingulate cortex, superior parietal cortex, pars opercularis, and superior frontal cortex of the right hemisphere (Figure 2). Given that the cortical thickness different between these brain areas, these areas were selected to perform further analyses.

\section{Cortical Thickness of the Moderate Group Compared With the HC Group}

The moderate group exhibited large areas (surface size $\left.>100 \mathrm{~mm}^{2}\right)$ of significant decreases $(p<0.001)$ in the

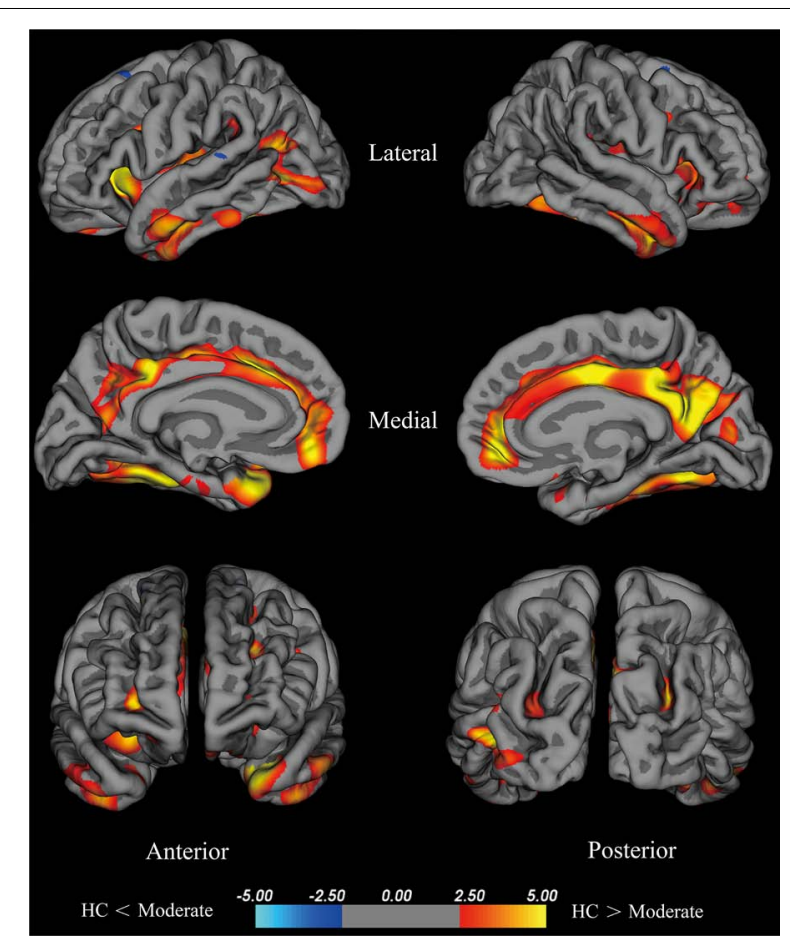

FIGURE 3 | Brain areas with significant cortical thickness differences between the $\mathrm{HC}$ group and the moderate group in the right and left hemispheres $(0<0.001)$. PD patients with $2.5 \leq \mathrm{H}-\mathrm{Y}$ stage $\geq 2$ were included in the moderate group. Red: $\mathrm{HC}>$ moderate, the closer the color is to yellow, the greater the difference. Blue: $\mathrm{HC}<$ moderate, the lighter the blue is, the larger the difference.

temporal pole, insula, fusiform cortex, isthmus cingulate cortex, lateral occipital cortex, middle temporal cortex, inferior parietal cortex, rostral middle frontal cortex, precentral cortex, inferior temporal gyrus, supra marginal gyrus, and superior parietal cortex of the left hemisphere, precuneus, medial orbitofrontal, fusiform cortex, postcentral gyrus, lateral orbitofrontal cortex, rostral middle frontal cortex, middle temporal cortex, superior parietal cortex, and caudal middle frontal cortex of the right hemisphere (Figure 3). We hypothesize that these differential areas are related to PD.

\section{Cortical Thickness of the Mild Group Compared With the HC Group}

The mild group exhibited significant reductions in cortical thickness in the left supra marginal gyrus $(p<0.001)$.

\section{Regression Analysis of Average Cortical Thickness}

There were statistically significant differences in the average cortical thickness of the following eight brain areas: the temporal pole $(p=0.005)$, fusiform gyrus $(p<0.001)$, insula of the left hemisphere $(p=0.004)$, fusiform gyrus $(p=0.001)$, isthmus cingulate cortex $(p=0.001)$, inferior temporal gyrus $(p=0.003)$, middle temporal cortex $(p=0.001)$ and posterior cingulate cortex of the right hemisphere $(p=0.001)$ among the HC, mild and moderate groups. After controlling for age, gender, and duration, a regression analysis of the UPDRS-III scores of PD patients 
TABLE 3 | Regression analysis of UPDRS-III scores and cortical thickness in different brain areas.

\begin{tabular}{lccc}
\hline Regions & \multicolumn{3}{c}{ UPDRS-III } \\
\cline { 2 - 4 } & Adjusted $\boldsymbol{R}^{\mathbf{2}}$ & $\boldsymbol{p}$-value & \multicolumn{1}{c}{$\boldsymbol{\beta}$} \\
\hline L fusiform gyrus & 0.290 & 0.049 & -0.227 \\
L temporal pole gyrus & 0.148 & 0.022 & -0.291 \\
R fusiform gyrus & 0.285 & 0.039 & -0.239 \\
\hline
\end{tabular}

with the cortical thickness of the left and right fusiform gyrus and the left temporal pole was performed, results are shown in Table 3, the cortical thickness of these brain areas were negatively correlated with the UPDRS-III scores.

\section{DISCUSSION}

The current study analyzed regions of cortical thinning and comprehensively assessed the relationship between cortical thinning and UPDRS-III by comparing $\mathrm{HC}$, mild $\mathrm{PD}$ and moderate PD groups. Our findings provide new insights into the pathogenesis and neuropathology of PD and may aid the development of new MRI biomarkers of disease progression.

Current limitations such as inadequate attention and difficulty diagnosing PD affect the treatment of the disease. Therefore, exploring the imaging features of PD patients can aid in the early diagnosis of PD. PD symptoms and disease severity are typically assessed using the UPDRS score and $\mathrm{H}-\mathrm{Y}$ staging; $\mathrm{H}-\mathrm{Y}$ staging $\leq 2.5$ is considered to be the early stage of PD (Chen et al., 2016). Cortical atrophy, amygdalar atrophy and cortical thinning in the fronto-temporal region of PD patients were specifically associated with PD degeneration (Ibarretxe-Bilbao et al., 2012). Age (Uribe et al., 2016), gender (Luders et al., 2006), disease duration (Madhyastha et al., 2015) are associated with the cortical thickness, which might reflect a mixed pathologic features, such as neuronal density (La Fougere et al., 2011), cerebral perfusion (Nobili et al., 2011; Madhyastha et al., 2015), degenerative pathologies including tau, $\alpha$-synuclein in PD patients (Ye et al., 2017). Deng et al. (2016b) found that the cortical morphological changes of brain regions (i.e., frontal, temporal, parietal, occipital and limbic lobes; cerebellum; caudate; and thalamus) were closely related to clinical symptoms of sporadic PD patients, including $\mathrm{H}-\mathrm{Y}$ stage and UPDRS-III scores. This finding is consistent with our finding that the PD patients exhibited significantly lower mean cortical thickness of the temporal pole, fusiform gyrus, insula of the left hemisphere and the fusiform gyrus, isthmus cingulate cortex, inferior temporal gyrus, middle temporal cortex and posterior cingulate cortex of the right hemisphere than the HC group. In addition, UPDRS-III scores in PD patients were negatively correlated with the left and right fusiform gyrus and left temporal pole, thus providing further evidence of MRI biomarkers for specific brain changes in the pathophysiology of PD motor dysfunction.

Many different methods have been used in early PD crosssectional imaging studies, and there are large variations in the structural changes of the cortex and WM. For example, changes in cortical thickness (Mak et al., 2015), GM volume (Jia et al., 2015) and cortical microstructure (Nurnberger et al., 2017), increased cortical and subcortical atrophy rate (Tessa et al., 2014) and loss of cortical gyrification (Sterling et al., 2016) have been reported, and some imaging signs even correlate with disease progression (Ibarretxe-Bilbao et al., 2011; Jubault et al., 2011). Another previously published study reported that disease stage in $\mathrm{PD}$ was associated with thinning of the medial frontal, posterior cingulate, precuneus, lateral occipital, temporal and dorsolateral prefrontal cortex (Zarei et al., 2013). In contrast to our study, which enrolled PD patients with $\mathrm{H}-\mathrm{Y}$ stage $\leq 2.5$, Zarei et al. divided PD patients into three groups: group 1 consisted of $\mathrm{PD}$ patients with $\mathrm{H}-\mathrm{Y}$ stage $\leq 2$, group 2 consisted of PD patients with $\mathrm{H}-\mathrm{Y}$ stage 2-4, and group 3 consisted of PD patients with $\mathrm{H}-\mathrm{Y}$ stage $>3$ (Zarei et al., 2013). In our study, the temporal cortex (including the temporal pole, fusiform gyrus of the left hemisphere, fusiform gyrus, inferior temporal gyrus, and middle temporal cortex of the right hemisphere), and the posterior cingulate cortex of the right hemisphere were consistent with Zarei et al.'s finding (Zarei et al., 2013). A pattern of GM changes has been suggested to occur in the mesial frontal cortex in PD patients with FOG. Pietracupa et al. (2017) found that PD patients with FOG exhibit significant changes in WM and cortical thinning of the mesial surface of the bilateral cerebral hemispheres. Vastik et al. (2017) found that FOG was associated with regional GM atrophy in the mesial frontal and cingulate cortices. Consistent with our results, the thickness of the posterior cingulate cortex and the temporal cortex were correlated with $\mathrm{H}-\mathrm{Y}$ stages. Previous research demonstrated that mirror movements were associated with bilateral overactivation of the precuneus/posterior cingulate cortex and the insula (Harel et al., 2013). Another study revealed that cortical thickness changes in the bilateral posterior cingulate cortex were correlated with a higher cognitive risk score (Ye et al., 2017). The results point to the likelihood that a decline in regional cerebral blood flow $(\mathrm{rCBF})$ in the posterior cingulate cortex is related to PD disease progression (Imamura et al., 2008; Nobili et al., 2011). Recent studies have found that decreased cortical thickness of the fusiform gyrus is related to cognitive impairment (Pagonabarraga et al., 2013; Zarei et al., 2013). Interestingly, temporal pole atrophy is consistent with the Braak classification of PD pathology (Potgieser et al., 2014). We believe that the combination of different MRI techniques and brain areas may contribute to improving evaluations of progressive brain involvement and may eventually reveal surrogate markers of disease progression.

Given that different $\mathrm{H}-\mathrm{Y}$ stages indicate different disease progression stages of $\mathrm{PD}$, different $\mathrm{H}-\mathrm{Y}$ stages exhibit different neuropathology, neuroimaging, and/or clinical evidence. Thus, imaging studies focusing on $\mathrm{PD}$ patients at different $\mathrm{H}-\mathrm{Y}$ stages can help understand the pathogenesis and disease progression of PD. NMS are extremely common throughout all stages of $\mathrm{PD}$ and are more critical to detect in the early stages of the disease, as NMS are closely related to the pathological process of $\mathrm{PD}$. In recent years, the value of recognizing NMS of $\mathrm{PD}$ for the early diagnosis of $\mathrm{PD}$ has been noted (Berg et al., 2015; Postuma et al., 2015). 
Therefore, better identifying NMS may increase the possibility of detecting PD biomarkers in the early stage of PD, and the development of novel MRI methods will provide further new imaging markers for the early diagnosis and treatment of PD. In a study of mid-sporadic PD patients, Deng et al. (2016a) reported that dysfunctions in some brain areas with cortical thinning are closely related to clinical symptoms, such as cognitive impairment, hallucinations, psychosis, depressed mood, anxious mood, apathy, sleep problems, and sexual desire disorder. Other studies have reported abnormal changes, such as cortical thinning (Danti et al., 2015; Uribe et al., 2016) and GM volume atrophy (Foo et al., 2017), in PD patients with cognitive impairment, whereas PD patients with RBD (Boucetta et al., 2016), depression (Huang et al., 2016; Luo et al., 2016), impulsivity (Pellicano et al., 2015) and NMS also exhibit cortical changes. Many NMS during the PD prodromal phase are clearly associated with the occurrence of PD and are clear risk factors. Therefore, cortical changes in PD patients associated with NMS have an important role in the early diagnosis of PD.

Numerous studies have confirmed that cognitive dysfunction and motor symptoms are correlated. In newly diagnosed PD patients, the severity of motor symptoms at the first visit correlates with cognitive impairment and is associated with an increased risk of developing MCI (Siciliano et al., 2017). The postural instability gait difficulty motor phenotype is a risk factor for the development of PD dementia (Muller et al., 2013). Dysfunction in the right dorsal medulla oblongata shell and core can account for association learning deficits in patients with leftsided PD (Huang et al., 2017). Other studies confirmed that progression on the $\mathrm{H}-\mathrm{Y}$ scale positively predicted the extent of cognitive impairment (Wu et al., 2012; Pedersen et al., 2013). However, other reports suggest no correlation between cognition and motor function. For example, Weintraub et al. (2015) did not identify a significant correlation between $\mathrm{H}-\mathrm{Y}$ classification and cognitive impairment using the MoCA scale, but the study was limited by a lack of effective neurocognitive scales. The fronto-temporo-parietal pattern of cortical thinning pattern does not associate with motor laterality (Danti et al., 2015). In the prodromal stage of $\mathrm{PD}$, a progressive process could occur from non-motor to motor performances until the disease becomes clinically evident, which is consistent with Braak's proposed mechanism. Therefore, PD imaging studies should be combined with studies on motor and NMS, especially RBD and cognitive dysfunction, to contribute to the diagnosis of early and even prodromal PD.

The main limitations of this study include three points. First, the sample size included in this study is relatively small, and

\section{REFERENCES}

Alves, G., Forsaa, E. B., Pedersen, K. F., Dreetz Gjerstad, M., and Larsen, J. P. (2008). Epidemiology of Parkinson's disease. J. Neurol. 255(Suppl. 5), 18-32. doi: 10.1007/s00415-008-5004-3

Berg, D., Postuma, R. B., Adler, C. H., Bloem, B. R., Chan, P., Dubois, B., et al. (2015). MDS research criteria for prodromal Parkinson's disease. Mov. Disord. 30, 1600-1611. doi: $10.1002 / \mathrm{mds} .26431$ we need to increase the sample size and conduct follow-up observations in subsequent studies. Second, further studies are needed to quantify potential differences between $\mathrm{PD}$ patients with $\mathrm{H}-\mathrm{Y} \leq 2.5$ and those with $\mathrm{H}-\mathrm{Y}>2.5$. Third, the study controlled for age, gender and duration to avoid confounding effects, but there may be confounding factors (e.g., years of education and cognitive score) that should be addressed in future studies.

In conclusion, the $\mathrm{HC}$, mild and moderate groups exhibited significant decreasing trends in the mean cortical thickness of the following eight brain areas: the temporal pole, fusiform gyrus, insula of the left hemisphere and fusiform gyrus, isthmus cingulate cortex, inferior temporal gyrus, middle temporal cortex and posterior cingulate cortex of the right hemisphere. The UPDRS-III scores in patients with early PD were correlated with cortical thickness of the left and right fusiform gyrus and left temporal pole. The results of this study provide a research direction for basic research aimed to better understand neuropathology in PD patients, contribute to the early diagnosis of $\mathrm{PD}$, and provide a new neuroimaging biomarker for $\mathrm{PD}$.

\section{AUTHOR CONTRIBUTIONS}

YG, KN, YZ, and LjW conceived and designed the study and drafted the manuscript. $\mathrm{BH}$ is responsible for performing MRI studies. MM is responsible for performing MRI data processing. MG is helped draft the manuscript. YG, ZH, LmW, and JZ conceived and coordinated the neurocognitive evaluation and helped with statistical analysis. YZ and $\mathrm{LjW}$ helped draft the manuscript and supervised the research project. All authors read and approved the final manuscript.

\section{FUNDING}

This work was supported by grants of National Natural Science Foundation of China (Nos. 81501112, 81671275, and 81471654), The National Key R\&D Program of China (No. 2017YFC1310200), and Guangdong Natural Science Foundation (Nos. 2016A030310327, 2017B030311015, and 2015A030313536).

\section{ACKNOWLEDGMENTS}

The authors thank all the subjects who kindly agreed to participate in this study.

Boucetta, S., Salimi, A., Dadar, M., Jones, B. E., Collins, D. L., and Dang-Vu, T. T. (2016). Structural brain alterations associated with rapid eye movement sleep behavior disorder in Parkinson's disease. Sci. Rep. 6:26782. doi: 10.1038/ srep 26782

Braak, H., Del Tredici, K., Rüb, U., De Vos, R. A. I., Jansen Steur, E. N. H., and Braak, E. (2003). Staging of brain pathology related to sporadic Parkinson's disease. Neurobiol. Aging 24, 197-211. doi: 10.1016/S0197-4580(02) 00065-9 
Chen, S., Chan, P., Sun, S., Chen, H., Zhang, B., Le, W., et al. (2016). The recommendations of Chinese Parkinson's disease and movement disorder society consensus on therapeutic management of Parkinson's disease. Transl. Neurodegener. 5:12. doi: 10.1186/s40035-016-0059-z

Danti, S., Toschi, N., Diciotti, S., Tessa, C., Poletti, M., Del Dotto, P., et al. (2015). Cortical thickness in de novo patients with Parkinson disease and mild cognitive impairment with consideration of clinical phenotype and motor laterality. Eur. J. Neurol. 22, 1564-1572. doi: 10.1111/ene.12785

Deng, X., Tang, C. Y., Zhang, J., Zhu, L., Xie, Z. C., Gong, H. H., et al. (2016a). The cortical thickness correlates of clinical manifestations in the mid-stage sporadic Parkinson's disease. Neurosci. Lett. 633, 279-289. doi: 10.1016/j.neulet.2016. 09.042

Deng, X., Zhou, M., Tang, C., Zhang, J., Zhu, L., Xie, Z., et al. (2016b). The alterations of cortical volume, thickness, surface, and density in the intermediate sporadic Parkinson's disease from the han population of Mainland China. Front. Aging Neurosci. 8:185. doi: 10.3389/fnagi.2016. 00185

Dorsey, E. R., Constantinescu, R., Thompson, J. P., Biglan, K. M., Holloway, R. G., Kieburtz, K., et al. (2006). Projected number of people with Parkinson disease in the most populous nations, 2005 through 2030. Neurology 68, 384-386. doi: 10.1212/01.wnl.0000247740.47667.03

Evans, J. R., Mason, S. L., Williams-Gray, C. H., Foltynie, T., Brayne, C., Robbins, T. W., et al. (2011). The natural history of treated Parkinson's disease in an incident, community based cohort. J. Neurol. Neurosurg. Psychiatry 82, 1112-1118. doi: 10.1136/jnnp.2011.240366

Fischl, B. (2012). FreeSurfer. Neuroimage 62, 774-781. doi: 10.1016/j.neuroimage. 2012.01.021

Fischl, B., and Dale, A. M. (2000). Measuring the thickness of the human cerebral cortex from magnetic resonance images. Proc. Natl. Acad. Sci. U.S.A. 97, 11050-11055. doi: 10.1073/pnas.200033797

Foo, H., Mak, E., Yong, T. T., Wen, M. C., Chander, R. J., Au, W. L., et al. (2017). Progression of subcortical atrophy in mild Parkinson's disease and its impact on cognition. Eur. J. Neurol. 24, 341-348. doi: 10.1111/ene. 13205

Harel, N., Poisson, A., Ballanger, B., Metereau, E., Redouté, J., Ibarolla, D., et al. (2013). A functional magnetic resonance imaging study of pathophysiological changes responsible for mirror movements in Parkinson's disease. PLoS One 8:e66910. doi: 10.1371/journal.pone.0066910

Hoehn, M. M., and Yahr, M. D. (1967). Parkinsonism: onset, progression and mortality. Neurology 17, 427-442. doi: 10.1212/WNL.17.5.427

Huang, P., Lou, Y., Xuan, M., Gu, Q., Guan, X., Xu, X., et al. (2016). Cortical abnormalities in Parkinson's disease patients and relationship to depression: a surface-based morphometry study. Psychiatry Res. 250, 24-28. doi: 10.1016/j. pscychresns.2016.03.002

Huang, P., Tan, Y. Y., Liu, D. Q., Herzallah, M. M., Lapidow, E., Wang, Y., et al. (2017). Motor-symptom laterality affects acquisition in Parkinson's disease: a cognitive and functional magnetic resonance imaging study. Mov. Disord. 32, 1047-1055. doi: $10.1002 / \mathrm{mds} .27000$

Ibarretxe-Bilbao, N., Junque, C., Marti, M. J., and Tolosa, E. (2011). Brain structural MRI correlates of cognitive dysfunctions in Parkinson's disease. J. Neurol. Sci. 310, 70-74. doi: 10.1016/j.jns.2011.07.054

Ibarretxe-Bilbao, N., Junque, C., Segura, B., Baggio, H. C., Marti, M. J., Valldeoriola, F., et al. (2012). Progression of cortical thinning in early Parkinson's disease. Mov. Disord. 27, 1746-1753. doi: 10.1002/mds. 25240

Imamura, K., Wada-Isoe, K., Kowa, H., Tanabe, Y., and Nakashima, K. (2008). The effect of donepezil on increased regional cerebral blood flow in the posterior cingulate cortex of a patient with Parkinson's disease dementia. Neurocase 14, 271-275. doi: 10.1080/13554790802269984

Jia, X., Liang, P., Li, Y., Shi, L., Wang, D., and Li, K. (2015). Longitudinal study of gray matter changes in Parkinson disease. AJNR Am. J. Neuroradiol. 36, 2219-2226. doi: 10.3174/ajnr.A4447

Jubault, T., Gagnon, J. F., Karama, S., Ptito, A., Lafontaine, A. L., Evans, A. C., et al. (2011). Patterns of cortical thickness and surface area in early Parkinson's disease. Neuroimage 55, 462-467. doi: 10.1016/j.neuroimage.2010. 12.043

Kalaitzakis, M. E., Graeber, M. B., Gentleman, S. M., and Pearce, R. K. B. (2008). Controversies over the staging of $\alpha$-synuclein pathology in
Parkinson's disease. Acta Neuropathol. 116, 125-128. doi: 10.1007/s00401-0080381-3

Kalia, L. V., and Lang, A. E. (2015). Parkinson's disease. Lancet 386, 896-912. doi: 10.1016/S0140-6736(14)61393-3

La Fougere, C., Grant, S., Kostikov, A., Schirrmacher, R., Gravel, P., Schipper, H. M., et al. (2011). Where in-vivo imaging meets cytoarchitectonics: the relationship between cortical thickness and neuronal density measured with high-resolution [18F] flumazenil-PET. Neuroimage 56, 951-960. doi: 10.1016/ j.neuroimage.2010.11.015

Luders, E., Narr, K. L., Thompson, P. M., Rex, D. E., Woods, R. P., Deluca, H., et al. (2006). Gender effects on cortical thickness and the influence of scaling. Hum. Brain Mapp. 27, 314-324. doi: 10.1002/hbm.20187

Luo, C., Song, W., Chen, Q., Yang, J., Gong, Q., and Shang, H. F. (2016). Cortical thinning in drug-naive Parkinson's disease patients with depression. J. Neurol. 263, 2114-2119. doi: 10.1007/s00415-016-8241-x

Madhyastha, T. M., Askren, M. K., Boord, P., Zhang, J., Leverenz, J. B., and Grabowski, T. J. (2015). Cerebral perfusion and cortical thickness indicate cortical involvement in mild Parkinson's disease. Mov. Disord. 30, 1893-1900. doi: $10.1002 / \mathrm{mds} .26128$

Mak, E., Su, L., Williams, G. B., Firbank, M. J., Lawson, R. A., Yarnall, A. J., et al. (2015). Baseline and longitudinal grey matter changes in newly diagnosed Parkinson's disease: ICICLE-PD study. Brain 138, 2974-2986. doi: 10.1093/ brain/awv211

Muller, M. L., Frey, K. A., Petrou, M., Kotagal, V., Koeppe, R. A., Albin, R. L., et al. (2013). beta-Amyloid and postural instability and gait difficulty in Parkinson's disease at risk for dementia. Mov. Disord. 28, 296-301. doi: 10.1002/mds.25213

Nobili, F., Arnaldi, D., Campus, C., Ferrara, M., De Carli, F., Brugnolo, A., et al. (2011). Brain perfusion correlates of cognitive and nigrostriatal functions in de novo Parkinson's disease. Eur. J. Nucl. Med. Mol. Imaging 38, 2209-2218. doi: 10.1007/s00259-011-1874-1

Nurnberger, L., Gracien, R. M., Hok, P., Hof, S. M., Rub, U., Steinmetz, H., et al. (2017). Longitudinal changes of cortical microstructure in Parkinson's disease assessed with T1 relaxometry. Neuroimage Clin. 13, 405-414. doi: 10.1016/j.nicl. 2016.12 .025

Pagonabarraga, J., Corcuera-Solano, I., Vives-Gilabert, Y., Llebaria, G., GarciaSanchez, C., Pascual-Sedano, B., et al. (2013). Pattern of regional cortical thinning associated with cognitive deterioration in Parkinson's disease. PLoS One 8:e54980. doi: 10.1371/journal.pone.0054980

Pedersen, K. F., Larsen, J. P., Tysnes, O. B., and Alves, G. (2013). Prognosis of mild cognitive impairment in early Parkinson disease: the Norwegian ParkWest study. JAMA Neurol. 70, 580-586. doi: 10.1001/jamaneurol.2013.2110

Pellicano, C., Niccolini, F., Wu, K., O’sullivan, S. S., Lawrence, A. D., Lees, A. J., et al. (2015). Morphometric changes in the reward system of Parkinson's disease patients with impulse control disorders. J. Neurol. 262, 2653-2661. doi: 10.1007/ s00415-015-7892-3

Pietracupa, S., Suppa, A., Upadhyay, N., Gianni, C., Grillea, G., Leodori, G., et al. (2017). Freezing of gait in Parkinson's disease: gray and white matter abnormalities. J. Neurol. 265, 52-62. doi: 10.1007/s00415-017-8654-1

Postuma, R. B., Berg, D., Stern, M., Poewe, W., Olanow, C. W., Oertel, W., et al. (2015). MDS clinical diagnostic criteria for Parkinson's disease. Mov. Disord. 30, 1591-1601. doi: 10.1002/mds.26424

Potgieser, A. R. E., Van Der Hoorn, A., Meppelink, A. M., Teune, L. K., Koerts, J., and De Jong, B. M. (2014). Anterior temporal atrophy and posterior progression in patients with Parkinson's disease. Neurodegener. Dis. 14, 125-132. doi: 10. $1159 / 000363245$

Siciliano, M., De Micco, R., Trojano, L., De Stefano, M., Baiano, C., Passaniti, C., et al. (2017). Cognitive impairment is associated with Hoehn and Yahr stages in early, de novo Parkinson disease patients. Parkinsonism Relat. Disord. 41, 86-91. doi: 10.1016/j.parkreldis.2017.05.020

Sterling, N. W., Wang, M., Zhang, L., Lee, E. Y., Du, G., Lewis, M. M., et al. (2016). Stage-dependent loss of cortical gyrification as Parkinson disease "unfolds". Neurology 86, 1143-1151. doi: 10.1212/WNL.0000000000 002492

Tessa, C., Lucetti, C., Giannelli, M., Diciotti, S., Poletti, M., Danti, S., et al. (2014). Progression of brain atrophy in the early stages of Parkinson's disease: a longitudinal tensor-based morphometry study in de novo patients without cognitive impairment. Hum. Brain Mapp. 35, 3932-3944. doi: 10.1002/hbm. 22449 
Uribe, C., Segura, B., Baggio, H. C., Abos, A., Marti, M. J., Valldeoriola, F., et al. (2016). Patterns of cortical thinning in nondemented Parkinson's disease patients. Mov. Disord. 31, 699-708. doi: 10.1002/mds. 26590

Vastik, M., Hok, P., Valosek, J., Hlustik, P., Mensikova, K., and Kanovsky, P. (2017). Freezing of gait is associated with cortical thinning in mesial frontal cortex. Biomed. Pap. Med. Fac. Univ. Palacky. Olomouc. Czech. Repub. 161, 389-396. doi: $10.5507 /$ bp. 2017.035

Weintraub, D., Simuni, T., Caspell-Garcia, C., Coffey, C., Lasch, S., Siderowf, A., et al. (2015). Cognitive performance and neuropsychiatric symptoms in early, untreated Parkinson's disease. Mov. Disord. 30, 919-927. doi: 10.1002/mds. 26170

Wu, Q., Chen, L., Zheng, Y., Zhang, C., Huang, L., Guo, W., et al. (2012). Cognitive impairment is common in Parkinson's disease without dementia in the early and middle stages in a Han Chinese cohort. Parkinsonism Relat. Disord. 18, 161-165. doi: 10.1016/j.parkreldis.2011.09.009

Ye, B. S., Jeon, S., Ham, J. H., Lee, J. J., Lee, J. M., Lee, H. S., et al. (2017). Dementiapredicting cognitive risk score and its correlation with cortical thickness in
Parkinson disease. Dement. Geriatr. Cogn. Disord. 44, 203-212. doi: 10.1159/ 000479057

Zarei, M., Ibarretxe-Bilbao, N., Compta, Y., Hough, M., Junque, C., Bargallo, N., et al. (2013). Cortical thinning is associated with disease stages and dementia in Parkinson's disease. J. Neurol. Neurosurg. Psychiatry 84, 875-881. doi: 10.1136/ jnnp-2012-304126

Conflict of Interest Statement: The authors declare that the research was conducted in the absence of any commercial or financial relationships that could be construed as a potential conflict of interest.

Copyright (c) 2018 Gao, Nie, Mei, Guo, Huang, Wang, Zhao, Huang, Zhang and Wang. This is an open-access article distributed under the terms of the Creative Commons Attribution License (CC BY). The use, distribution or reproduction in other forums is permitted, provided the original author(s) and the copyright owner(s) are credited and that the original publication in this journal is cited, in accordance with accepted academic practice. No use, distribution or reproduction is permitted which does not comply with these terms. 